\title{
To stent or stent-graft? It depends
}

\author{
James I. Fann, MD
}

\section{See related article on pages 3003-11.}

Endovascular treatment of thoracic aortic disorders has upended much of what we had convinced ourselves was the right way to do things in the open surgical past. For those keeping track, it has been more than 2 decades since the first endovascular stent-graft was deployed in the treatment of thoracic aortic aneurysms. It has also been that long since the first endovascular approach to direct stenting of compromised branches from a dissected thoracic aorta. Although the medical management of uncomplicated acute type B dissection is preferred, once complications develop the optimal approach becomes less well-defined, mainly because of the variability of end-organ ischemia and injury, the more severe of which is associated with a correspondingly worse outcome.

In their study, Massmann and colleagues ${ }^{1}$ address a difficult to treat subset of patients presenting with acute type B dissection-namely, those with the complication of visceral ischemia. To define perhaps the optimal therapeutic approach requires one to set the baseline by thoughtfully evaluating the outcomes of that approach. In this case, 14 patients with gastrointestinal malperfusion complicating acute type $\mathrm{B}$ dissection are managed with a specific focus on reestablishing flow to the visceral branches through endovascular stenting, which is in contradistinction to thoracic aortic stentgrafting. More important than the study limitations relating to the small number of patients in a retrospective analysis is the thought process that pervades the procedural description, making a case for addressing the anatomic and physiologic variability and arguing for an individualized approach. Insightfully, Massmann and colleagues ${ }^{1}$ describe a considered approach to the early treatment of these patients; in fact, in many cases, the initial approach is directed at the descending thoracic aorta followed, if necessary, by intervening on the affected visceral vessel. So, not surprisingly, the optimal approach relies on optimal planning.

From the Department of Cardiothoracic Surgery, Stanford University Medical Center, Stanford, Calif.

Disclosures: Author has nothing to disclose with regard to commercial support.

Received for publication Sept 24, 2014; accepted for publication Sept 25, 2014; available ahead of print Oct 18, 2014.

Address for reprints: James I. Fann, MD, Department of Cardiothoracic Surgery, Stanford University Medical Center, 300 Pasteur Dr, Falk CV Research Center, Stanford, CA 94305 (E-mail: jfann@stanford.edu).

J Thorac Cardiovasc Surg 2014;148:3012-3

0022-5223/\$0.00

Published by Elsevier Inc. on behalf of The American Association for Thoracic Surgery

http://dx.doi.org/10.1016/j.jtcvs.2014.09.091
Because not all patients demonstrated angiographic narrowing of the visceral vessel in this series, one should not underestimate the impact of proximal true-lumen compression on visceral perfusion. The first step of endovascular treatment addressed inflow issues and stenting of the compressed proximal true lumen with a self-expandable stent. But such an approach can have unintended consequences or unfulfilled expectations, as some required further stenting of the infrarenal aorta to provide flow to the inferior mesenteric artery and iliac arteries. After aortic stenting, persistently compromised visceral arteries in a small fraction of patients were directly treated with balloon-expandable stents. Also important, and mentioned in passing, the commonly used fenestration was not in of itself of substantive benefit in their experience.

Recognizing that this endovascular approach is not entirely new, as other investigators have successfully intervened on similar patient subsets in the past, Massmann and colleagues ${ }^{1}$ make an effort to document the outcome beyond the early perioperative period, particularly as it relates to patient survival, late complications, and aortic remodeling. Clearly, the aortic pathology and technical constraints are dynamic, and what one sees early may not be what it is later. In particular, at 1 week of follow-up, there were 4 cases of collapsed or compressed aortic stents, which were unexpected and not wholly accounted for. The collapsed stents were balloon dilated, but collapse recurred 1 month later. One of the stents fractured at 3 months of follow-up; however, the cause of stent collapse in the others remained elusive and might be related to lack of complete stent expansion, with resultant less radial force or persistent pulsatile flow in the false lumen. Regarding the fate of the aorta, Massmann and colleagues ${ }^{1}$ appreciated no increase in the descending thoracic aortic diameter during follow-up; however, 1 patient had an increased abdominal aortic diameter. Even with thoracic aortic stenting, the false lumen remained patent without thrombosis. Although 2 patients subsequently required aortic valve and ascending aortic replacement for ascending aortic aneurysm, none needed intervention on the descending aorta. Fortunately, and notwithstanding the mechanical complications, the patients remained free of symptoms during medium-term follow-up. Nonetheless, it is unclear as to the long-term outcome and whether further intervention will be necessary.

Massmann and colleagues ${ }^{1}$ acknowledge that their experience does not prove that their approach is the best approach. It does, however, support a compelling argument 
that uncovered stenting has a role, perhaps a very good one, in the primary treatment of these complicated cases. Just as they are thoughtful and considered in planning their approach, readers likewise should be thoughtful in interpreting their findings. Perhaps a longer follow-up of a larger number of patients and the use of this technique at other institutions will provide the needed support. No doubt more experience will help us define the best techniqueuntil then, to stent or stent-graft? It depends.

\section{Reference}

1. Massmann A, Kunihara T, Fries P, Schneider G, Buecker A, Schäfers HJ. Uncovered stent implantation in complicated acute aortic dissection type B. J Thorac Cardiovasc Surg. 2014;148:3003-11. 